\title{
Synthesis and structure of alkylzinc 3,5-diphenylpyrazolates: Dramatic influence of steric and solvent effects
}

Szymon Komorski, ${ }^{\dagger}$ Michał K. Leszczyński, ${ }^{\ddagger}$ Iwona Justyniak, ${ }^{\dagger}$ and Janusz Lewiński*, ${ }^{\dagger, *}$

${ }^{\dagger}$ Warsaw University of Technology, Faculty of Chemistry, Noakowskiego 3, 00-664 Warsaw, Poland

${ }^{\star}$ Polish Academy of Sciences, Institute of Physical Chemistry, Kasprzaka 44/52, 01-224 Warsaw, Poland

Contents:

I. Synthesis of compounds 1-3

II. X-ray structure determination

III. ${ }^{1} \mathrm{H}$ NMR and DOSY NMR

IV. Molecular weight estimation based on diffusivity measurements 


\section{Synthesis of compounds 1-3}

General Considerations. All manipulations were conducted in a $\mathrm{N}_{2}$ atmosphere using standard Schlenk techniques. Solvents were dried and distilled from sodium-potassium alloy and benzophenone prior to use. All reagents were purchased from commercial vendors and used in a dry $\mathrm{N}_{2}$ atmosphere.

Synthesis of $\left[{ }^{\mathrm{t}} \mathrm{BuZn}\left(\mathrm{pz}^{\mathrm{Ph} 2}\right)\right]_{3} \cdot$ hexane $\left(\mathbf{1}_{3^{\bullet}}\right.$ hexane): A slurry of 3,5-diphenylpyrazole (440mg, $\left.2 \mathrm{mmol}\right)$ in toluene $(10 \mathrm{ml})$ was cooled to $-15^{\circ} \mathrm{C}$ and afterwards a $1 \mathrm{M}$ solution of ${ }^{t} \mathrm{Bu}_{2} \mathrm{Zn}(2 \mathrm{ml}, 2 \mathrm{mmol})$ in toluene was added dropwise. The reaction mixture was left stirring for 30 minutes at this temperature, followed by another 30 minutes of stirring at ambient temperature. Compound $\mathbf{1}$ was obtained after concentration of mother solution followed by addition of hexane and crystallization at $0^{\circ} \mathrm{C}$; isolated yield c.a. $621 \mathrm{mg}(84 \%)$. C,H,N analysis (\%) calcd for $\mathrm{C}_{63} \mathrm{H}_{74} \mathrm{~N}_{6} \mathrm{Zn}_{3}$ : C 68.08, H 6.71, N7.56; found C $67.89, \mathrm{H} 6.56, \mathrm{~N} 7.66$. Upon dissolution of the crystals of $\mathbf{1}_{\mathbf{3}}$ hexane in toluene- $d 8$, two aggregates were observed in the ${ }^{1} \mathrm{H}$ NMR spectrum $-\mathbf{1}_{2}$ and $\mathbf{1}_{3}$ (see Fig. S6 for NMR DOSY analysis) with a relative ratio of ca. $1: 5$ respectively. ${ }^{1} \mathrm{H}$ NMR (toluene- $d 8,25^{\circ} \mathrm{C}$ ) aggregate $\mathbf{1}_{\mathbf{2}}: \delta 7.64(\mathrm{~d}, \mathrm{~J}=7.0 \mathrm{~Hz}$ 4H), $7.29(\mathrm{t}, \mathrm{J}=7.4 \mathrm{~Hz}, 5 \mathrm{H}), 6.70(\mathrm{~s}, 1 \mathrm{H}), 0.73(\mathrm{~s}, 9 \mathrm{H})$; aggregate $\mathbf{1}_{3}: 7.39(\mathrm{~d}, \mathrm{~J}=7.4 \mathrm{~Hz}, 4 \mathrm{H}), 7.16(\mathrm{t}$, $\mathrm{J}=7.4 \mathrm{~Hz}, 4 \mathrm{H}), 7.08(\mathrm{~d}, \mathrm{~J}=7.4 \mathrm{~Hz}, 2 \mathrm{H}), 6.56(\mathrm{~s}, 1 \mathrm{H}), 0.69(\mathrm{~s}, 9 \mathrm{H})$. Due to significant overlapping in the aromatic region, not all of the signals could be observed separately for the two aggregates.

Synthesis of [( $\left.\left.\mathbf{E t}_{2} \mathbf{Z n}_{3}\left(\mathbf{p z}^{\mathrm{Ph} 2}\right)\right)_{4}\right]$ (2): A slurry of 3,5-diphenylpyrazole ( 440mg, $\left.2 \mathrm{mmol}\right)$ in toluene (10 $\mathrm{ml})$ was cooled to $-15^{\circ} \mathrm{C}$ and afterwards a $2 \mathrm{M}$ solution of $\mathrm{Et}_{2} \mathrm{Zn}(0.75 \mathrm{ml}, 1.5 \mathrm{mmol})$ in hexane was added dropwise. The reaction mixture was left stirring for 30 minutes at this temperature, followed by another 30 minutes of stirring at ambient temperature, upon which a crystalline solid deposited. Afterwards hexane $(3 \mathrm{ml})$ was added and the mixture was cooled to $0^{\circ} \mathrm{C}$ and left at this temperature overnight. The X-Ray suitable crystals were obtained through dissolution of that precipitate in hot toluene followed by addition of hexane and crystallization at ambient temperature; isolated yield c.a. $435 \mathrm{mg}$ (77\%). C,H,N analysis (\%) calcd for $\mathrm{C}_{64} \mathrm{H}_{54} \mathrm{~N}_{8} \mathrm{Zn}_{3}$ : C 67.95, H4.81, N 9.90; found C 67.98, H4.81, N 9.89. ${ }^{1} \mathrm{H}$ NMR (toluene- $\left.d 8300 \mathrm{MHz}, 25^{\circ} \mathrm{C}, \mathrm{ppm}\right): \delta 7.61-7.25(\mathrm{~m}, 16 \mathrm{H}), 6.34(\mathrm{~s}, 2 \mathrm{H}), 0.45$ (t, $\mathrm{J}=8.0 \mathrm{~Hz}, 3 \mathrm{H}),-0.32(\mathrm{q}, \mathrm{J}=7.9 \mathrm{~Hz}, 2 \mathrm{H})$

Synthesis of $\left[\left(\mathbf{E t}_{2} \mathbf{Z n}_{3}\left(\mathbf{p z}^{\mathrm{Ph} 2}\right)\right)_{4}\right] \cdot \mathbf{P h M e}\left(2^{\prime} \cdot \mathbf{P h M e}\right)$ : A slurry of 3,5-diphenylpyrazole ( 440mg, 2 $\mathrm{mmol})$ in toluene $(10 \mathrm{ml})$ was cooled to $-15^{\circ} \mathrm{C}$ and afterwards a $2 \mathrm{M}$ solution of $\mathrm{Et}_{2} \mathrm{Zn}(0.75 \mathrm{ml}, 1.5$ $\mathrm{mmol}$ ) in hexane was added dropwise. The reaction mixture was left stirring for 30 minutes at this temperature, followed by another 30 minutes of stirring at ambient temperature, upon which a crystalline solid deposited. Afterwards hexane $(3 \mathrm{ml})$ was added and the mixture was cooled to $0^{\circ} \mathrm{C}$ and left at this temperature overnight. The X-Ray suitable crystals were obtained through dissolution of that precipitate in hot toluene followed by addition of hexane and crystallization at $-15^{\circ} \mathrm{C}$. isolated

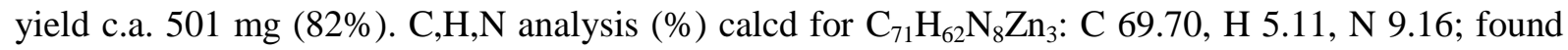
C $69.68 \mathrm{H} 5.12 \mathrm{~N} 9.16 .{ }^{1} \mathrm{H}$ NMR (toluene- $d 8300 \mathrm{MHz}, 25^{\circ} \mathrm{C}, \mathrm{ppm}$ ): $\delta$ 7.61-7.25 (m, 16H), 6.34 (s, $2 \mathrm{H}), 0.45(\mathrm{t}, \mathrm{J}=8.0 \mathrm{~Hz}, 3 \mathrm{H}),-0.32(\mathrm{q}, \mathrm{J}=7.9 \mathrm{~Hz}, 2 \mathrm{H})$

Synthesis of [(EtZn( $\left.\left.\mathbf{p z}^{\mathrm{Ph} 2}\right)\right)_{2}(\boldsymbol{\mu}$-THF)] (3): A solution of 3,5-diphenylpyrazole ( 440mg, 2 mmol) in THF $(10 \mathrm{ml})$ was cooled to $-15^{\circ} \mathrm{C}$ and afterwards a $2 \mathrm{M}$ solution of $\mathrm{Et}_{2} \mathrm{Zn}(1 \mathrm{ml}, 2 \mathrm{mmol})$ in hexane was added dropwise. The reaction mixture was left stirring for 30 minutes at this temperature, followed by another 30 minutes of stirring at ambient temperature. Compound $\mathbf{3}$ was obtained after concentration of mother solution followed by addition of hexane and crystallization at $-15^{\circ} \mathrm{C}$; isolated yield c.a. $439 \mathrm{mg}(63 \%)$. C,H,N analysis (\%) calcd for $\mathrm{C}_{38} \mathrm{H}_{40} \mathrm{~N}_{4} \mathrm{O}_{1} \mathrm{Zn}_{2}$ : C 65.25, H 5.76, N 8.01; found 
C 65.29, H 5.83, N 7.91. ${ }^{1} \mathrm{H}$ NMR (toluene- $\left.d 8300 \mathrm{MHz}, 25^{\circ} \mathrm{C}, \mathrm{ppm}\right): \delta 7.74-7.64(\mathrm{~m}, 4 \mathrm{H}), 7.39-$ $7.20(\mathrm{~m}, 6 \mathrm{H}), 6.69(\mathrm{~s}, 1 \mathrm{H}), 0.81(\mathrm{t}, \mathrm{J}=8.1 \mathrm{~Hz}, 3 \mathrm{H}), 0.12(\mathrm{q}, \mathrm{J}=8.0 \mathrm{~Hz}, 2 \mathrm{H})$.

\section{X-ray structure determination}

The crystals were selected under Paratone-N oil, mounted on the nylon loops and positioned in the cold stream on the diffractometer. The X-ray data for complexes 1, 2 and $\mathbf{3}$ were collected on a Nonius Kappa CCD diffractometer ${ }^{1}$ using graphite monochromated $\operatorname{MoK} \alpha$ radiation $(\lambda=0.71073 \AA)$. The unit cell parameters were determined from ten frames, then refined on all data. The data were processed with DENZO and SCALEPACK (HKL2000 package). ${ }^{2}$ The X-ray data for complex $\mathbf{2}^{\prime} \cdot \mathbf{P h C H}_{3}$ were collected at 100(2)K on a SuperNova Agilent diffractometer using MoKa radiation ( $\lambda$ $=0.71073 \AA$ ). The data were processed with CrysAlisPro. ${ }^{3}$ The structure was solved by direct methods using the SHELXS-97 program and was refined by full matrix least-squares on $\mathrm{F}^{2}$ using the program SHELXL-97. ${ }^{4}$ All non-hydrogen atoms were refined with anisotropic displacement parameters. Hydrogen atoms were added to the structure model at geometrically idealized coordinates and refined as riding atoms. Crystallographic data (excluding structure factors) for the structure reported in this paper have been deposited with the Cambridge Crystallographic Data Centre as supplementary publication. Copies of the data can be obtained free of charge on application to CCDC, 12 Union Road, Cambridge CB21EZ, UK (fax: (+44)1223-336-033; e-mail: deposit@ccdc.cam.ac.uk).

Crystal data for 1•0.5 $\mathbf{C}_{6} \mathbf{H}_{14} ; \mathrm{C}_{60} \mathrm{H}_{67} \mathrm{~N}_{6} \mathrm{Zn}_{3}: M=1068.31$, crystal dimensions $0.36 \times 0.24 \times 0.10 \mathrm{~mm}^{3}$, triclinic, space group $P-1$ (no. 2), $a=12.2480(6) \AA, b=13.4400(8) \AA, c=17.9650(12) \AA, \alpha=$ 88.007(3) ${ }^{\circ}, \beta=74.811(4)^{\circ}, \gamma=70.468(3)^{\circ}, U=2685.2(3) \AA^{3}, Z=2, F(000)=1118, D_{\mathrm{c}}=1.321 \mathrm{~g}$ $\mathrm{cm}^{-3}, T=100(2) \mathrm{K}, \mu(\mathrm{Mo}-\mathrm{K} \alpha)=1.373 \mathrm{~mm}^{-1}$, Nonius Kappa-CCD diffractometer, $\theta_{\max }=27.61^{\circ}, R 1=$ $0.0483, w R 2=0.1048$ for all data, $R 1=0.0420, w R 2=0.1011$ for 8278 reflections with $I_{o}>2 \sigma\left(I_{o}\right)$. The goodness-of-fit on $\mathrm{F}^{2}$ was equal 1.093. A weighting scheme $w=\left[\sigma^{2}\left(F_{\mathrm{o}}{ }^{2}+(0.0418 P)^{2}+3.1964 P\right]^{-}\right.$ ${ }^{1}$ where $P=\left(F_{\mathrm{o}}{ }^{2}+2 F_{\mathrm{c}}{ }^{2}\right) / 3$ was used in the final stage of refinement. The residual electron density = $+0.47 /-0.85 \mathrm{e}^{-3}$. CCDC -1463243 .

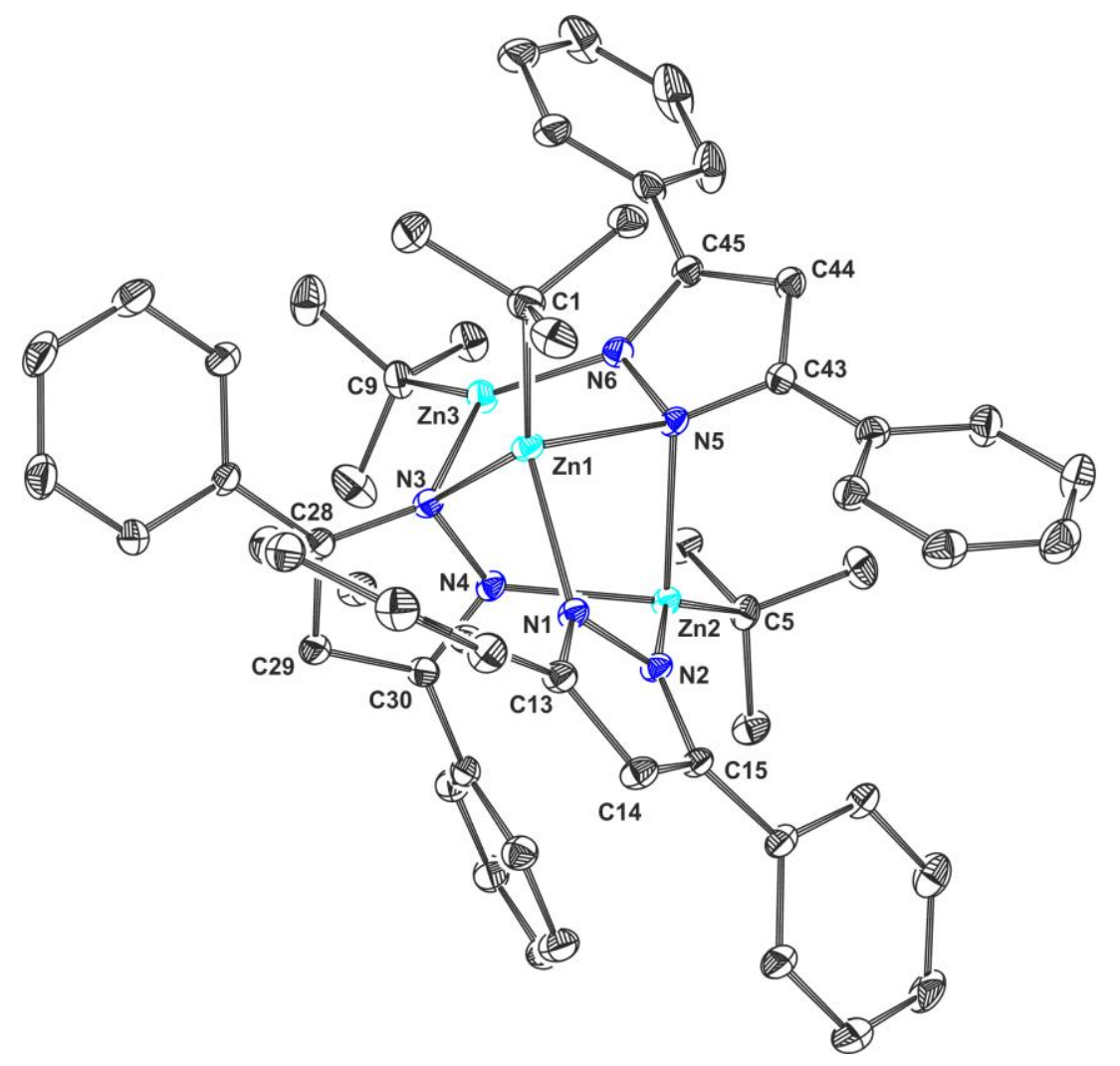


Figure S1. Molecular structure of 1 with thermal ellipsoids set at $35 \%$ probability. Hydrogen atoms have been omitted for clarity.

Table S1. Selected bond lengths ( $\AA$ ) and angles (deg) for $\mathbf{1}$.

$\begin{array}{llll}\text { Zn1 }-\mathrm{N} 1 & 2.022(2) & \mathrm{C} 1-\mathrm{Zn} 1-\mathrm{N} 1 & 127.33(11) \\ \mathrm{Zn} 1-\mathrm{N} 3 & 2.164(2) & \mathrm{C} 1-\mathrm{Zn} 1-\mathrm{N} 3 & 135.17(12) \\ \mathrm{Zn} 1-\mathrm{N} 5 & 2.309(2) & \mathrm{C} 1-\mathrm{Zn} 1-\mathrm{N} 5 & 106.93(10) \\ \mathrm{Zn} 1-\mathrm{C} 1 & 2.016(3) & \mathrm{C} 5-\mathrm{Zn} 2-\mathrm{N} 2 & 143.75(12) \\ \mathrm{Zn} 2-\mathrm{N} 2 & 2.022(2) & \mathrm{C} 5-\mathrm{Zn} 2-\mathrm{N} 4 & 116.32(11) \\ \mathrm{Zn} 2-\mathrm{N} 4 & 2.122(3) & \mathrm{C} 5-\mathrm{Zn} 2-\mathrm{N} 5 & 108.53(10) \\ \mathrm{Zn} 2-\mathrm{N} 5 & 2.242(2) & \mathrm{C} 9-\mathrm{Zn} 3-\mathrm{N} 3 & 123.05(11) \\ \mathrm{Zn} 2-\mathrm{C} 5 & 2.000(3) & \mathrm{C} 9-\mathrm{Zn} 3-\mathrm{N} 6 & 144.60(10) \\ \text { Zn3-N3 } & 2.179(2) & \mathrm{N} 3-\mathrm{Zn} 1-\mathrm{N} 1 & 92.25(9) \\ \mathrm{Zn} 3-\mathrm{N} 6 & 1.961(3) & \mathrm{N} 3-\mathrm{Zn} 1-\mathrm{N} 5 & 86.77(9) \\ \text { Zn3-C9 } & 1.975(3) & & \\ \end{array}$

Crystal data for $2 ; \mathrm{C}_{64} \mathrm{H}_{54} \mathrm{~N}_{8} \mathrm{Zn}_{3}: M=1131.26$, crystal dimensions $0.28 \times 0.18 \times 0.10 \mathrm{~mm}^{3}$, triclinic, space group $P-1$ (no. 2), $a=10.8470(3) \AA, b=16.0840(4) \AA, c=16.3890(5) \AA, \alpha=$ $70.2190(10)^{\circ}, \beta=85.270(2)^{\circ}, \gamma=75.359(2)^{\circ}, U=2603.18(13) \AA^{3}, Z=2, F(000)=1168, D_{\mathrm{c}}=$ $1.443 \mathrm{~g} \mathrm{~cm}^{-3}, T=100(2) \mathrm{K}, \mu(\mathrm{Mo}-\mathrm{K} \alpha)=1.422 \mathrm{~mm}^{-1}$, Nonius Kappa-CCD diffractometer, $\theta_{\max }=$ $26.46^{\circ}, R 1=0.0746, w R 2=0.1706$ for all data, $R 1=0.1064, w R 2=0.1840$ for 7910 reflections with $I_{o}>2 \sigma\left(I_{o}\right)$. The goodness-of-fit on $\mathrm{F}^{2}$ was equal 1.059. A weighting scheme $w=\left[\sigma^{2}\left(F_{\mathrm{o}}^{2}+\right.\right.$ $\left.(0.0418 P)^{2}+3.1964 P\right]^{-1}$ where $P=\left(F_{\mathrm{o}}{ }^{2}+2 F_{\mathrm{c}}{ }^{2}\right) / 3$ was used in the final stage of refinement. The residual electron density $=+1.21 /-1.05 \mathrm{e}^{-3}$. CCDC -1463244 .

Compound 2 formed a twinned crystal with partially superimposed reciprocal lattices. The transformation matrix of indices of the twin components for crystals was determined roughly as: $\begin{array}{lllllllll}-1.000 & 0.000 & 0.000 & \mid-0.730 & 0.847 & 0.307 & -0.365 & 0.923 & -0.847\end{array}$

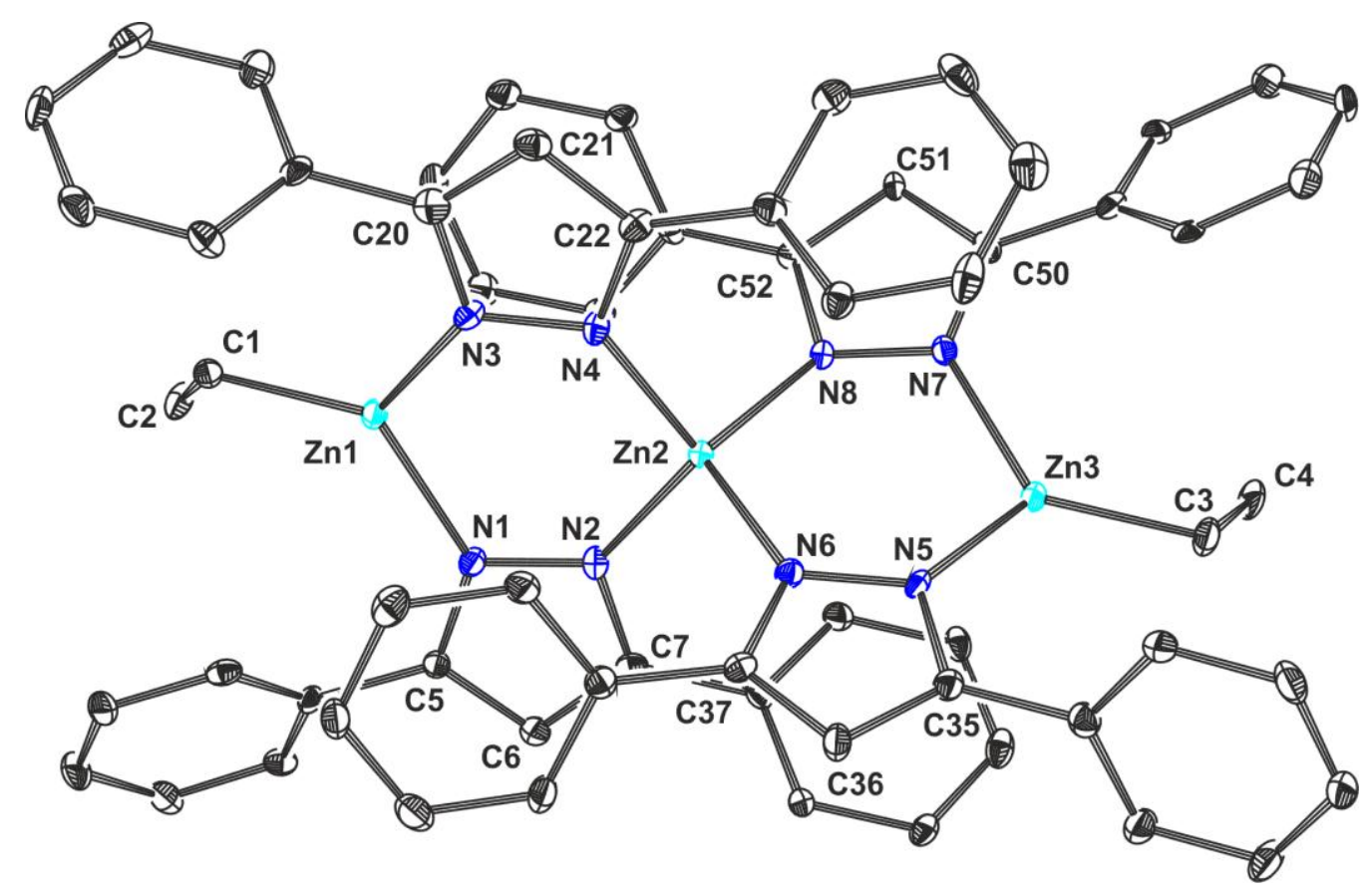


Figure S2. Molecular structure of 2 with thermal ellipsoids set at $35 \%$ probability. Hydrogen atoms have been omitted for clarity.

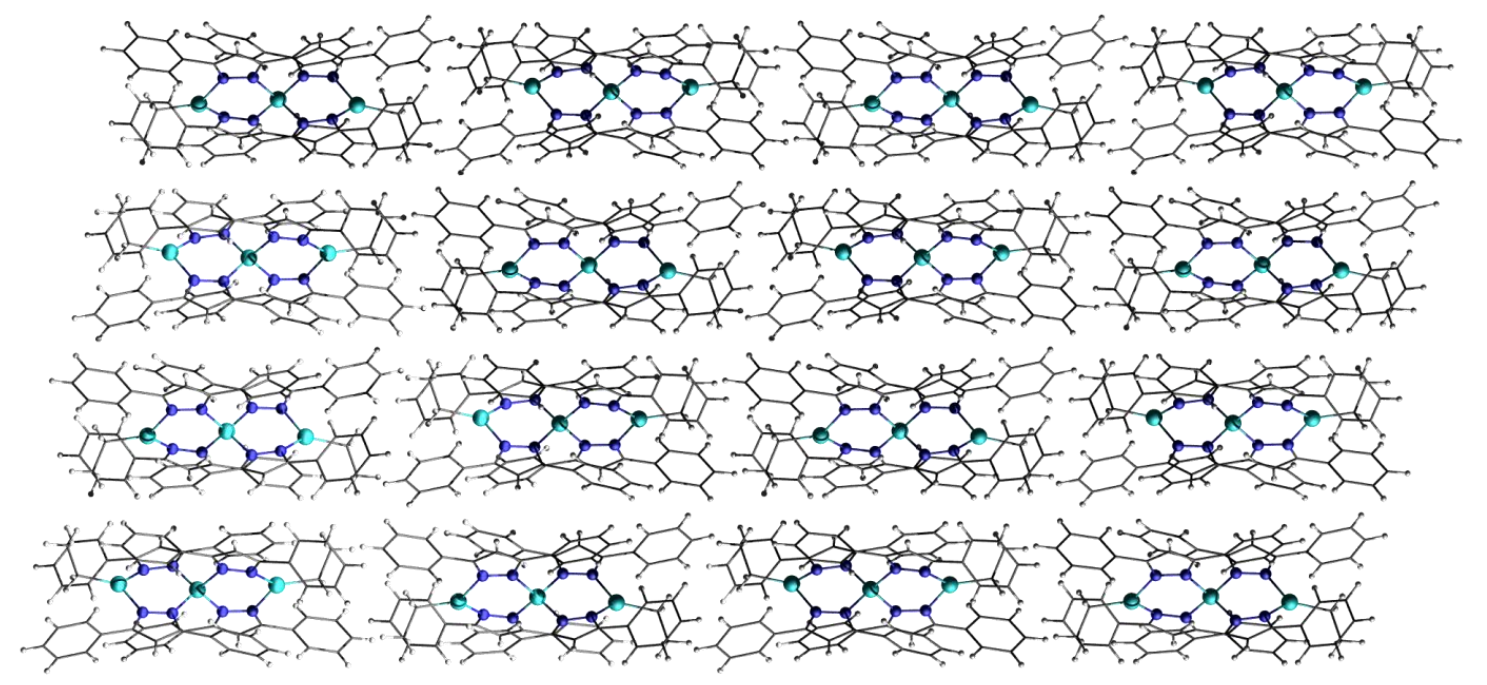

Figure S3. A view of the crystal structure of compound 2 along $a$ axis.

Table S2. Selected bond lengths $(\AA)$ and angles (deg) for $\mathbf{2}$.

\begin{tabular}{llll}
\hline $\mathrm{Zn} 1-\mathrm{C} 1$ & $1.993(6)$ & $\mathrm{C} 1-\mathrm{Zn} 1-\mathrm{N} 1$ & $128.8(2)$ \\
$\mathrm{Zn} 1-\mathrm{N} 1$ & $2.022(5)$ & $\mathrm{C} 1-\mathrm{Zn} 1-\mathrm{N} 3$ & $123.3(2)$ \\
$\mathrm{Zn} 1-\mathrm{N} 3$ & $2.011(5)$ & $\mathrm{N} 1-\mathrm{Zn} 1-\mathrm{N} 3$ & $107.5(2)$ \\
$\mathrm{Zn} 2-\mathrm{N} 2$ & $2.005(5)$ & $\mathrm{N} 2-\mathrm{Zn} 2-\mathrm{N} 8$ & $118.0(2)$ \\
$\mathrm{Zn} 2-\mathrm{N} 4$ & $1.985(5)$ & $\mathrm{N} 2-\mathrm{Zn} 2-\mathrm{N} 4$ & $109.1(2)$ \\
$\mathrm{Zn} 2-\mathrm{N} 6$ & $1.981(5)$ & $\mathrm{N} 4-\mathrm{Zn} 2-\mathrm{N} 6$ & $115.9(2)$ \\
$\mathrm{Zn} 2-\mathrm{N} 8$ & $2.004(5)$ & $\mathrm{N} 6-\mathrm{Zn} 2-\mathrm{N} 8$ & $109.0(2)$ \\
$\mathrm{Zn} 3-\mathrm{C} 3$ & $1.975(6)$ & $\mathrm{C} 3-\mathrm{Zn} 3-\mathrm{N} 5$ & $127.2(2)$ \\
$\mathrm{Zn} 3-\mathrm{N} 5$ & $2.002(5)$ & $\mathrm{C} 3-\mathrm{Zn} 3-\mathrm{N} 7$ & $125.9(2)$ \\
$\mathrm{Zn} 3-\mathrm{N} 7$ & $2.014(5)$ & $\mathrm{N} 5-\mathrm{Zn} 3-\mathrm{N} 7$ & $106.50(19)$ \\
& & & \\
\hline
\end{tabular}

Crystal data for $\mathbf{2}^{\prime} \cdot \mathbf{P h C H}_{3} ; \mathrm{C}_{71} \mathrm{H}_{62} \mathrm{~N}_{8} \mathrm{Zn}_{3}: M=1223.40$, crystal dimensions $0.28 \times 0.16 \times 0.08$ $\mathrm{mm}^{3}$, orthorhombic, space group $P 21212$ (no. 18), $a=17.1278(4) \AA, b=15.5508(4) \AA, c=$ 10.8341(3) $\AA, U=2885.67(13) \AA^{3}, Z=2, F(000)=1268, D_{\mathrm{c}}=1.408 \mathrm{~g} \mathrm{~cm}^{-3}, T=100(2) \mathrm{K}, \mu(\mathrm{Mo}-$ $\mathrm{K} \alpha)=1.289 \mathrm{~mm}^{-1}$, SuperNova Agilent diffractometer, $\theta_{\max }=27.48^{\circ}, R 1=0.0568, w R 2=0.0775$ for all data, $R 1=0.0448, w R 2=0.0715$ for 5363 reflections with $I_{o}>2 \sigma\left(I_{o}\right)$. The goodness-of-fit on $\mathrm{F}^{2}$ was equal 1.048. A weighting scheme $w=\left[\sigma^{2}\left(F_{\mathrm{o}}{ }^{2}+(0.0418 P)^{2}+3.1964 P\right]^{-1}\right.$ where $P=$ $\left(F_{\mathrm{o}}{ }^{2}+2 F_{\mathrm{c}}{ }^{2}\right) / 3$ was used in the final stage of refinement. The residual electron density $=+0.55 /-$ $0.40 \mathrm{e}^{-3}$. CCDC -1463245 . 


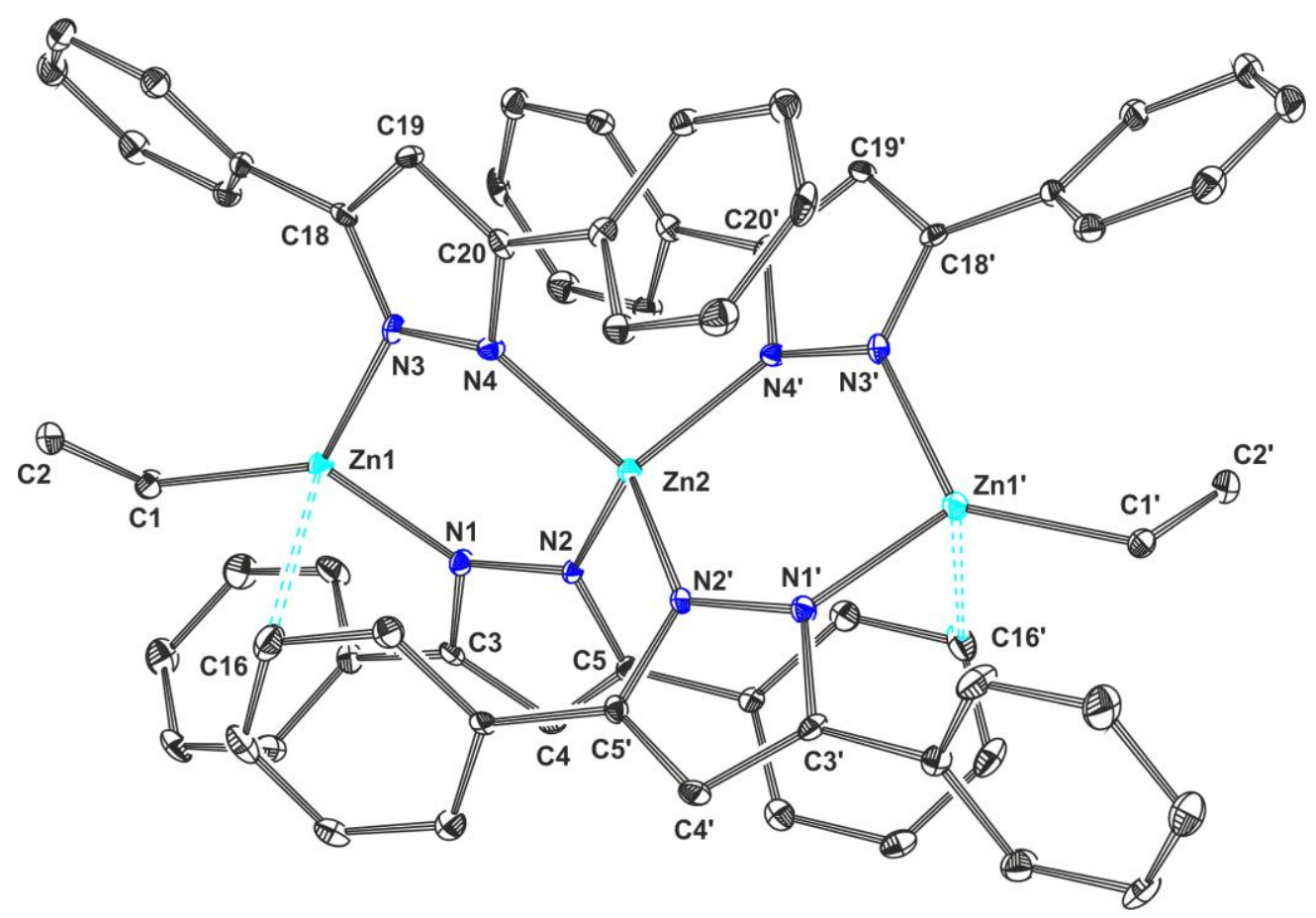

Figure S4. Molecular structure of $\mathbf{2}^{\prime} \cdot \mathbf{P h C H}_{3}$ with thermal ellipsoids set at $35 \%$ probability. Hydrogen atoms have been omitted for clarity. . The $\mathrm{Zn}-\pi$ interaction motif has been emphasized by cyan dashed bonds (Zn1-C16 and Zn1'-C16'). Symmetry codes: $(-x+2,-y+2, z),(-x+2,-y+1, z)$.

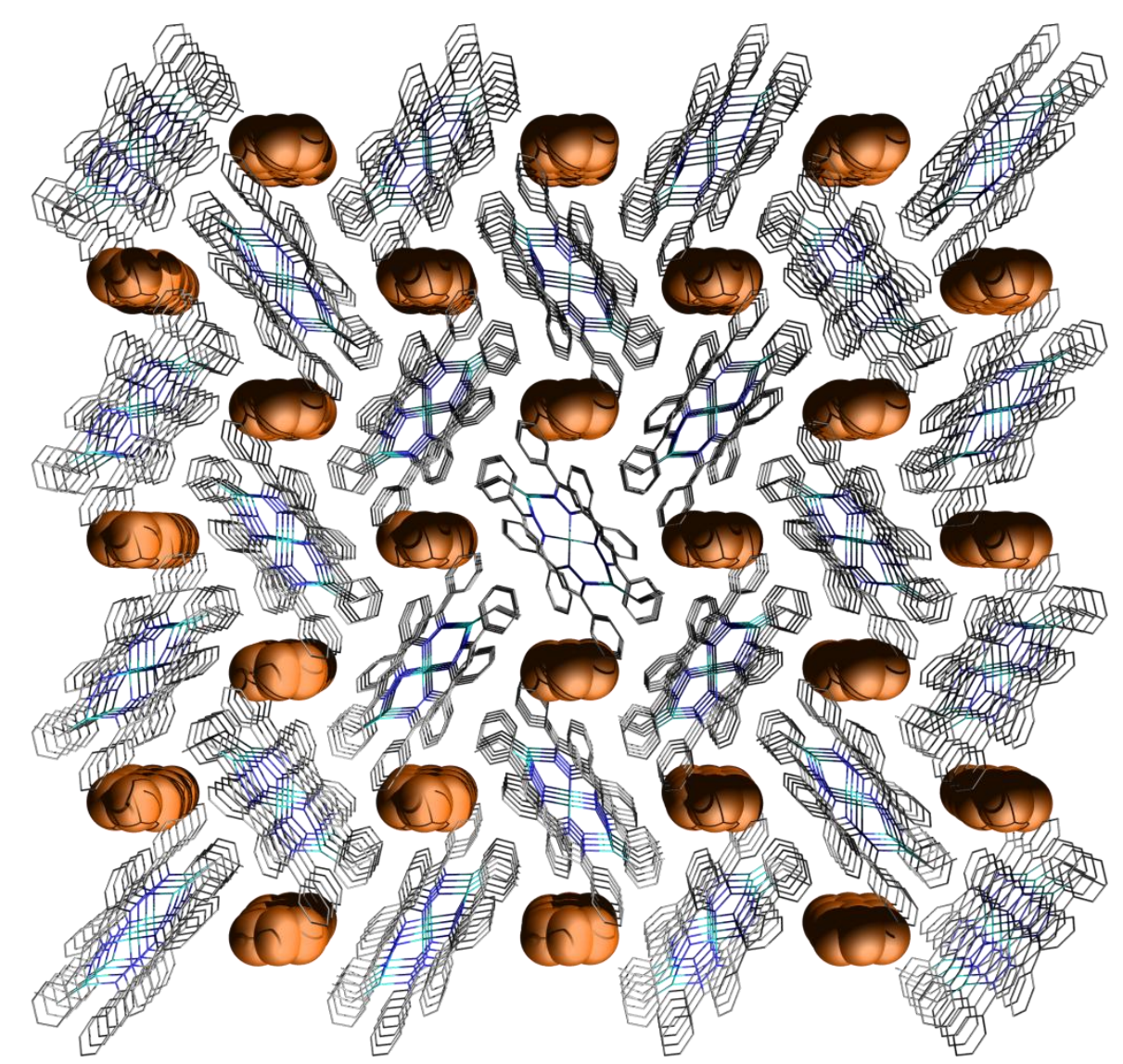

Figure S5. A perspective view of the crystal structure of $2 \cdot \mathrm{PhMe}$ along the $c$ axis with space-filling representation of the solvent molecules. 
Table S3. Selected bond lengths $(\AA)$ and angles $(\mathrm{deg})$ for $\mathbf{2}^{\prime} \cdot \mathbf{P h C H}$.

$\begin{array}{llll}\text { Zn1 }-\mathrm{C} 1 & 1.967(4) & \mathrm{C} 1-\mathrm{Zn} 1-\mathrm{N} 1 & 129.36(13) \\ \mathrm{Zn} 1-\mathrm{N} 1 & 1.996(3) & \mathrm{C} 1-\mathrm{Zn} 1-\mathrm{N} 3 & 126.07(13) \\ \mathrm{Zn} 1-\mathrm{C} 16 & 3.119(3) & \mathrm{N} 1-\mathrm{Zn} 1-\mathrm{N} 3 & 104.50(11) \\ \mathrm{Zn} 1-\mathrm{N} 3 & 2.008(3) & \mathrm{N} 2-\mathrm{Zn} 2-\mathrm{N} 4 & 107.58(12) \\ \mathrm{Zn} 2-\mathrm{N} 2 & 2.005(2) & \mathrm{N} 2-\mathrm{Zn} 2-\mathrm{N} 2 & 103.91(14) \\ \mathrm{Zn} 2-\mathrm{N} 4 & 2.017(3) & \mathrm{N} 4-\mathrm{Zn} 2-\mathrm{N} 4 & 101.06(16)\end{array}$

Crystal data for 3; $\mathrm{C}_{38} \mathrm{H}_{40} \mathrm{~N}_{4} \mathrm{OZn}_{2}: M=699.48$, crystal dimensions $0.32 \times 0.20 \times 0.12 \mathrm{~mm}^{3}$, monoclinic, space group $C 2 / \mathrm{c}$ (no. 15), $a=25.830(2) \AA, b=7.9420(6) \AA, c=18.0693(17) \AA, \beta=$ 107.332(8) ${ }^{\circ}, U=3538.4(5) \AA^{3}, Z=4, F(000)=1456, D_{\mathrm{c}}=1.390 \mathrm{~g} \mathrm{~cm}^{-3}, T=293(2) \mathrm{K}, \mu(\mathrm{Mo}-$ $\mathrm{K} \alpha)=1.390 \mathrm{~mm}^{-1}$, Nonius Kappa-CCD diffractometer, $\theta_{\max }=26.37^{\circ}, R 1=0.0478, w R 2=$ 0.0957 for all data, $R 1=0.0366, w R 2=0.0900$ for 2992 reflections with $I_{o}>2 \sigma\left(I_{o}\right)$. The goodness-of-fit on $\mathrm{F}^{2}$ was equal 1.052. A weighting scheme $w=\left[\sigma^{2}\left(F_{\mathrm{o}}{ }^{2}+(0.0418 P)^{2}+3.1964 P\right]^{-}\right.$ ${ }^{1}$ where $P=\left(F_{\mathrm{o}}{ }^{2}+2{F_{\mathrm{c}}}^{2}\right) / 3$ was used in the final stage of refinement. The residual electron density $=+0.42 /-0.21 \mathrm{e}^{-3}$. CCDC -1463246 .

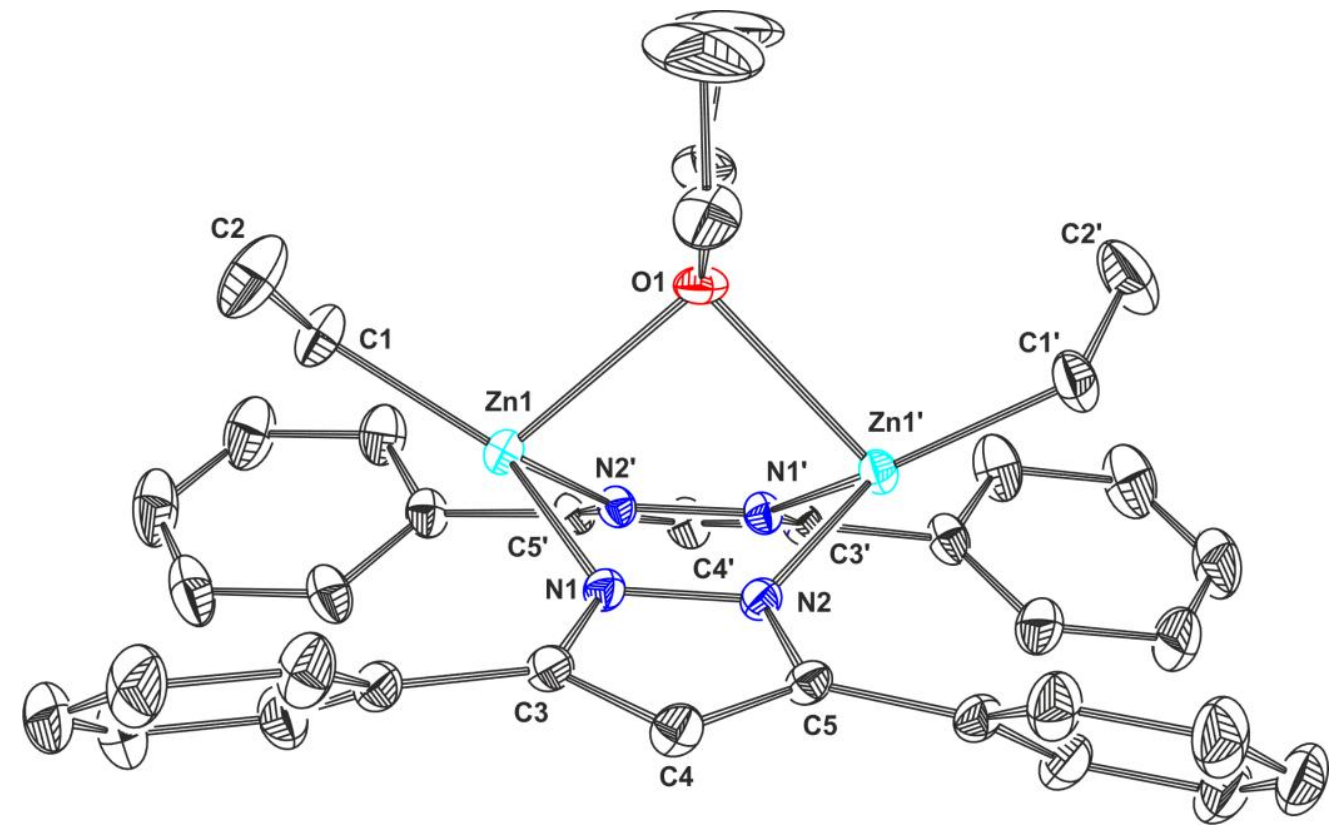

Figure S6. Molecular structure of $\mathbf{3}$ with thermal ellipsoids set at 30\% probability. Hydrogen atoms have been omitted for clarity. Symmetry codes: $(-x, y,-z+1 / 2)$.

Table S4. Selected bond lengths $(\AA)$ and angles (deg) for 3.

$\begin{array}{llll}\mathrm{Zn} 1-\mathrm{C} 1 & 1.956(3) & \mathrm{C} 1-\mathrm{Zn} 1-\mathrm{O} 1 & 106.83(13) \\ \mathrm{Zn} 1-\mathrm{N} 1 & 2.0091(19) & \mathrm{N} 1-\mathrm{Zn} 1-\mathrm{O} 1 & 85.96(6) \\ \mathrm{Zn} 1-\mathrm{N} 2 & 2.0258(19) & \mathrm{O} 1-\mathrm{Zn} 1-\mathrm{N} 2 & 85.46(6) \\ \mathrm{Zn} 1-\mathrm{O} 1 & 2.3452(16) & & \end{array}$




\section{III. ${ }^{1} \mathrm{H}$ NMR and DOSY NMR}

${ }^{1} \mathrm{H}$ NMR and DOSY NMR spectra of $\mathbf{1}_{3} \bullet$ hexane in toluene- $d 8$ at $25^{\circ} \mathrm{C}$

$$
\begin{aligned}
& 1_{2}-\left[{ }^{t} \mathrm{BuZn}\left(\mathrm{pz}^{\mathrm{Ph} 2}\right)\right]_{2} \\
& 1_{3}-\left[{ }^{t} \mathrm{BuZn}\left(\mathrm{pz}^{\mathrm{Ph} 2}\right)\right]_{3} \\
& \wedge \text { - residual hexane } \\
& *-\text { toluene- } \\
& \& \text { - } \text { silicone grease }^{-}
\end{aligned}
$$
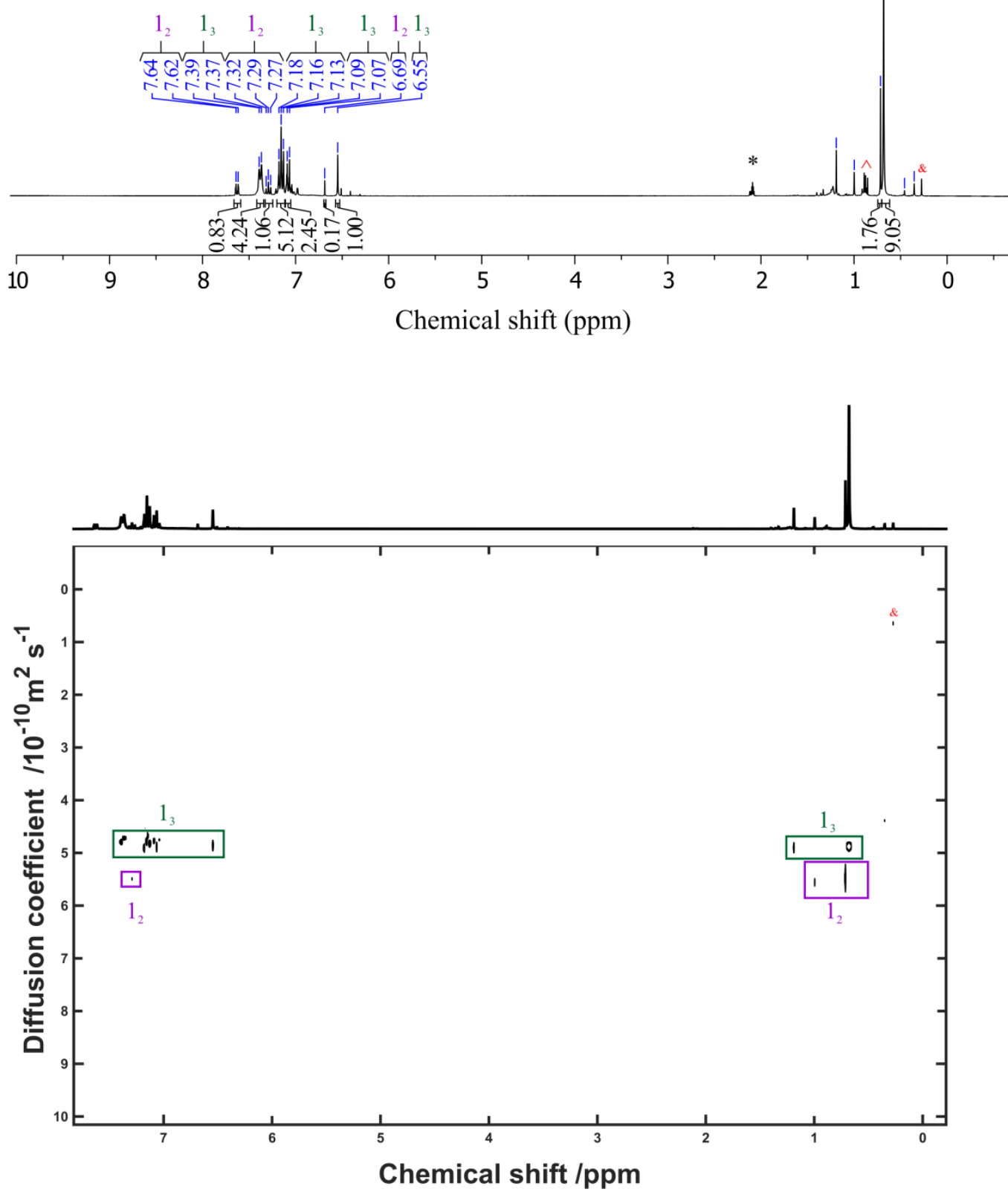

Figure S7. ${ }^{1} \mathrm{H}$ NMR and DOSY NMR spectra of $\mathbf{1}_{\mathbf{3}}$ 'hexane in toluene- $d 8$ at $25^{\circ} \mathrm{C}$ 
${ }^{1} \mathrm{H}$ NMR and DOSY NMR spectra of 2 in toluene- $d 8$ at $25^{\circ} \mathrm{C}$
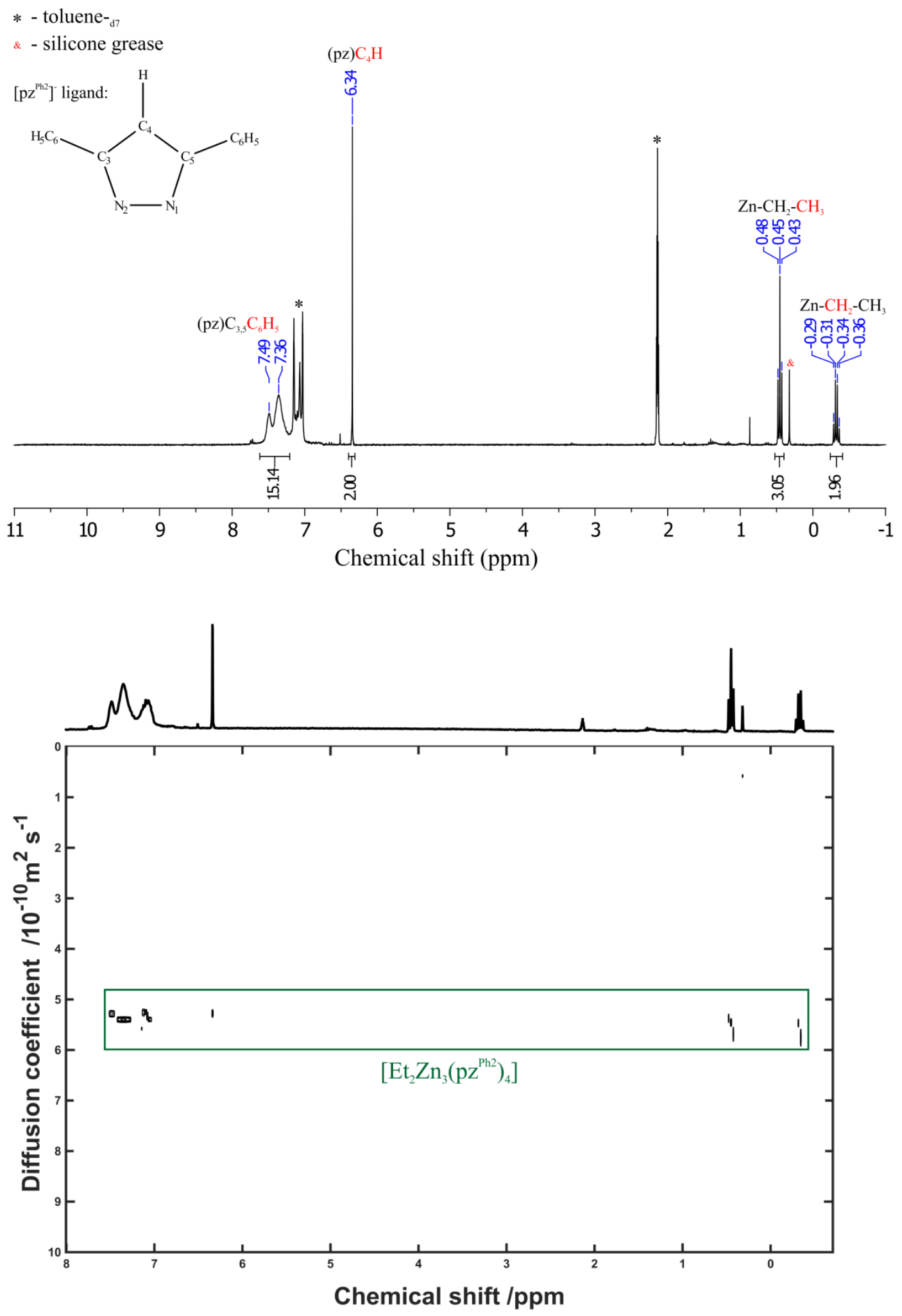

Figure S8. ${ }^{1} \mathrm{H}$ NMR and DOSY NMR spectra of $\mathbf{2}$ in toluene- $d 8$ at $25^{\circ} \mathrm{C}$ 
${ }^{1} \mathrm{H}$ NMR and DOSY NMR spectra of $\mathbf{3}$ in toluene- $d 8$ at $25^{\circ} \mathrm{C}$
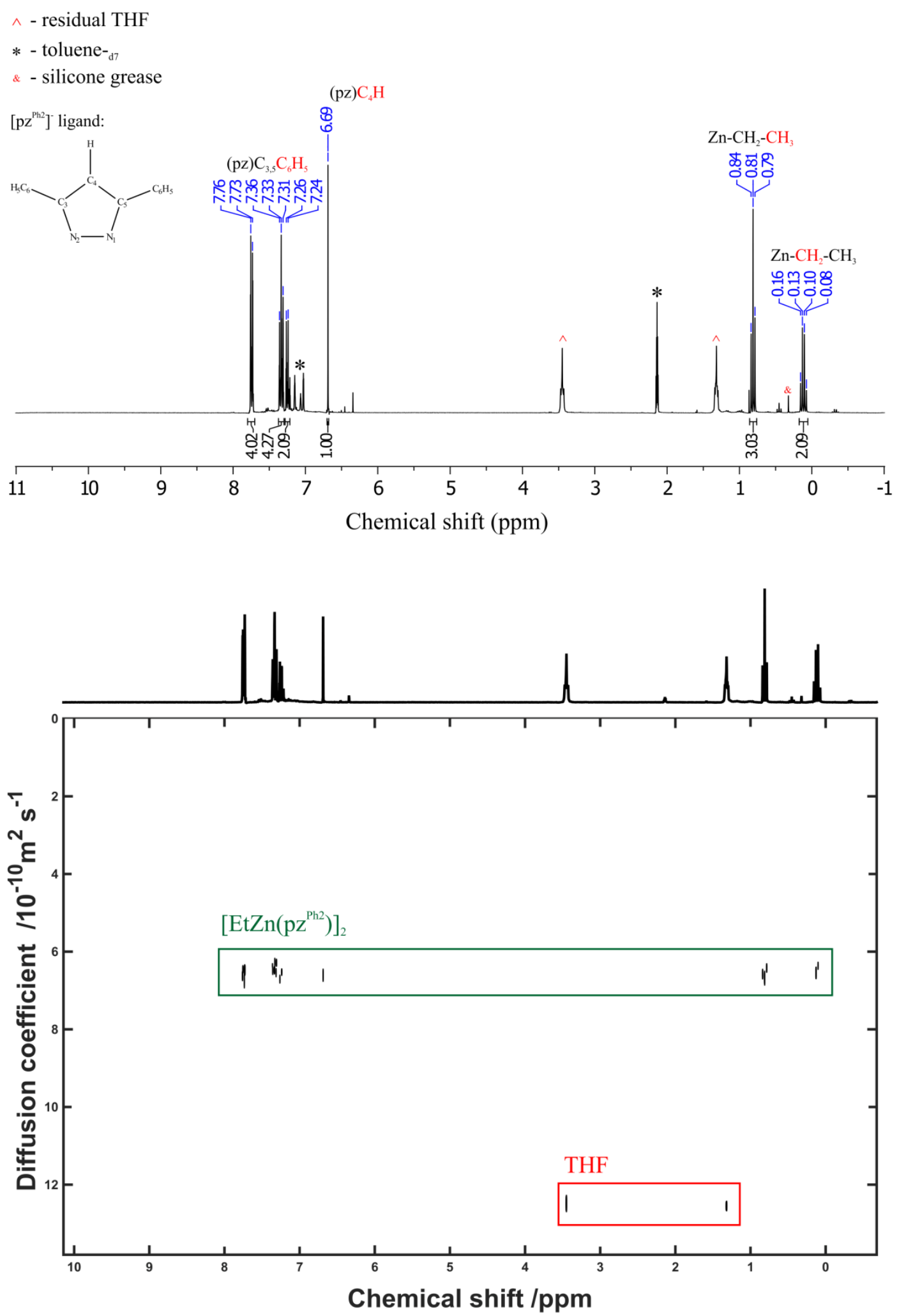

Figure S9. ${ }^{1} \mathrm{H}$ NMR and DOSY NMR spectra of $\mathbf{3}$ in toluene- $d 8$ at $25^{\circ} \mathrm{C}$ 


\section{Diffusivity measurement}

Application of Diffusion Ordered Spectroscopy (DOSY), which has happened to become a very routine NMR technique lately, provided us with a reliable evidence of approximate size of the species existing in the solution. As already discussed in antecedent works ${ }^{5,6}$ molecular weight can be straightforwardly obtained by combining Stokes-Einstein equation $D=\frac{k T}{6 \pi \rho r_{H}}$ and the relationship between molecular weight $M$ and molar radius, which reads $M=\frac{k T}{6 \pi \eta r_{H}}=\frac{4 \pi \rho N_{A} r_{M}}{3}$, where $\mathrm{r}_{\mathrm{H}}$ and $r_{M}$ are hydrodynamic and molar radii respectively, $\eta$ is viscosity $(6,02 \mathrm{E}-04 \mathrm{~Pa} \cdot \mathrm{s})$ and $\rho\left(0,932 \mathrm{~kg} / \mathrm{m}^{3}\right)$ is density of the liquid. It has also been shown that $r_{H}$ and $r_{M}$ are much alike for small molecules which is the case in our system. DOSY measurement was performed in dry and degassed toluene- $d 8$ as we exploited the fact that the physical properties of the diluted solution, namely density and viscosity, deviate only slightly from properties of the pure solvent. ${ }^{5}$ Raw data was processed with powerful DOSY Toolbox which is extensively described in its author's paper. ${ }^{7}$

The MWs calculated using DOSY derived diffusion coefficients for compounds 2 and $\mathbf{3}$ in Tables S6 and $\mathbf{S 7}$ show inconsistency with the solid state crystal structures and ${ }^{1} \mathrm{H}$ NMR spectra. The observed (ca. 50\% ) difference between the expected solid state structure MW and the calculated MWs can be explained by much greater deviation from the spherical shape of compounds $\mathbf{2}$ and $\mathbf{3}$ in contrast to $\mathbf{1}$.

Table S5.

\begin{tabular}{|c|c|c|c|}
\hline $\begin{array}{c}\text { Peak shift } \\
{[\mathrm{ppm}]}\end{array}$ & $\begin{array}{c}\mathrm{D} \\
{\left[10^{10} \cdot \mathrm{m}^{2} \cdot \mathrm{s}^{-1}\right]}\end{array}$ & $\begin{array}{c}\text { Rh } \\
{[\AA]}\end{array}$ & $\begin{array}{c}\text { MW } \\
{\left[\mathrm{g} \cdot \mathrm{mol}^{-1}\right]}\end{array}$ \\
\hline 7.64 & 5.693 & 6.37 & 608 \\
\hline 7.38 & 4.885 & 7.43 & 962 \\
\hline 7.29 & 5.080 & 7.14 & 856 \\
\hline 7.16 & 4.872 & 7.45 & 970 \\
\hline 7.08 & 4.925 & 7.37 & 939 \\
\hline 6.69 & 5.788 & 6.27 & 579 \\
\hline 6.55 & 4.871 & 7.45 & 971 \\
\hline 0.72 & 5.622 & 6.45 & 631 \\
\hline 0.68 & 5.015 & 7.23 & 890 \\
\hline
\end{tabular}

Calculated FW of $\mathbf{1}_{\mathbf{2}}=682$

Calculated FW of $\mathbf{1}_{\mathbf{3}}=1023$

Table S6.

\begin{tabular}{|c|c|c|c|}
\hline $\begin{array}{c}\text { Peak shift } \\
{[\mathrm{ppm}]}\end{array}$ & $\begin{array}{c}\mathrm{D} \\
{\left[10^{10} \cdot \mathrm{m}^{2} \cdot \mathrm{s}^{-1}\right]}\end{array}$ & $\begin{array}{c}\mathrm{Rh} \\
{[\AA]}\end{array}$ & $\begin{array}{c}\mathrm{MW} \\
{\left[\mathrm{g} \cdot \mathrm{mol}^{-1}\right]}\end{array}$ \\
\hline 7.49 & 5.24 & 6.92 & 778 \\
\hline 7.36 & 5.37 & 6.67 & 723 \\
\hline 6.34 & 5.64 & 6.42 & 624 \\
\hline 0.45 & 5.68 & 6.38 & 642 \\
\hline-0.32 & 5.59 & 6.49 & 610 \\
\hline
\end{tabular}

Calculated FW of $\mathbf{2}=1129$ 
Table S7.

\begin{tabular}{|c|c|c|c|}
\hline $\begin{array}{c}\text { Peak shift } \\
{[\mathrm{ppm}]}\end{array}$ & $\begin{array}{c}\mathrm{D} \\
{\left[10^{10} \cdot \mathrm{m}^{2} \cdot \mathrm{s}^{-1}\right]}\end{array}$ & $\begin{array}{c}\mathrm{Rh} \\
{[\AA]}\end{array}$ & $\mathrm{MW}\left[\mathrm{g} \cdot \mathrm{mol}^{-1}\right]$ \\
\hline 7.75 & 6.76 & 5.38 & 363 \\
\hline 7.33 & 6.57 & 5.52 & 395 \\
\hline 7.25 & 6.50 & 5.59 & 408 \\
\hline 6.69 & 6.52 & 5.57 & 405 \\
\hline 0.81 & 6.88 & 5.27 & 344 \\
\hline 0.12 & 6.84 & 5.30 & 351 \\
\hline
\end{tabular}

Calculated FW of $\mathbf{3}=698$

Calculated FW of $\left[\mathrm{EtZn}\left(\mathrm{pz}^{\mathrm{Ph} 2}\right)\right]_{2}=626$

\footnotetext{
${ }^{1}$ KappaCCD Software; Nonius B.V.: Delft, The Netherlands, 1998.

${ }^{2}$ Z. Otwinowski, W. Minor, Methods Enzymol. 1997, 276, 307.

${ }^{3}$ Agilent Technologies, CrysAlisPro, Version 1.171.35.21b.

${ }^{4}$ G. M. Sheldrick, Acta Cryst. 2008, 64A, 112-122.

${ }^{5}$ Li, D.; Kagan, G.; Hopson, R.; Williard, P. G. J. Am. Chem. Soc. 2009, 131, 5627-5634.

${ }^{6}$ Macchioni, A.; Ciancaleoni, G.; Zuccaccia, C.; Zuccaccia, D.; Chem. Soc. Rev. 2008, 37, 479-489.

${ }^{7}$ Nilsson, M.; J. Magn. Res. 2009, 2, 296-302.
} 